\title{
BANACH ALGEBRA ELEMENTS WHOSE SPECTRA INTERSECT $\mathbf{R}^{+}$
}

\author{
by F. F. BONSALL and A. C. THOMPSON \\ (Received 9th March 1973)
}

1

Let $A$ denote a complex Banach algebra with unit, $\operatorname{Inv}(A)$ the set of invertible elements of $A, \operatorname{Sp}(a)$ and $r(a)$ the spectrum and spectral radius respectively of an element $a$ of $A$. Let $\Gamma$ denote the set of elements of $A$ whose spectra contain non-negative real numbers, i.e.

$$
\Gamma=\left\{a \in A: \operatorname{Sp}(a) \cap \mathbf{R}^{+} \neq \varnothing\right\} .
$$

Yood (4) has observed that $\Gamma$ is an example of a set $Q$ satisfying

(i) $a \in Q, t \geqq 0 \Rightarrow t a \in Q$,

(ii) $a \in Q, r(a)<1 \Rightarrow a(1-a)^{-1} \in Q$,

(iii) $-1 \notin \bar{Q}$,

and has proved that every subset $Q$ of $A$ satisfying (i), (ii), (iii) is contained in $\Gamma$.

We show that the conclusion $Q \subset \Gamma$ also holds if (ii) is replaced by the purely algebraic condition:

(iv) $a \in Q \Rightarrow a+a^{2} \in Q$.

Our main theorem (Theorem 7) goes further than this and shows that the function $z(1-z)^{-1}$ in condition (ii) can be replaced by any member of a certain class of functions, this class containing both the functions $z(1-z)^{-1}$ and $z+z^{2}$.

It is natural to ask whether (ii) can be replaced by the condition:

$$
a \in Q \Rightarrow a^{2} \in Q \text {. }
$$

An example showing that this is not sufficient, and indeed that the semigroup condition

$$
a, b \in Q \Rightarrow a b \in Q
$$

is not sufficient, is given by the semigroup $Q=\left\{\rho e^{i \theta}: \rho \geqq 0, \theta=0,2 \pi / 3,4 \pi / 3\right\}$ in the algebra $\mathbf{C}$.

We prove in Theorem 1 that a subset $P$ of $\operatorname{Inv}(A)$ is contained in $\Gamma$ if it satisfies: $a \in P, t>0 \Rightarrow t a \in P ; a \in P, r\left(a^{-1}\right)<1 \Rightarrow a-1 \in P ;-1 \notin \bar{P}$. This gives Yood's theorem as an immediate corollary, and is one of the ingredients in the proof of Theorem 7. The other main ingredient is an iteration procedure previously used in the study of type 2 semi-algebras of non-negative continuous functions (1). 
We also consider in detail the relationship between the conditions (ii) and (iv); and end with two applications of our results.

\section{2}

Theorem 1. Let $P$ be a subset of $\operatorname{Inv}(A)$ such that

(i) $a \in P, t>0 \Rightarrow t a \in P$,

(ii) $a \in P, r\left(a^{-1}\right)<1 \Rightarrow a-1 \in P$,

(iii) $-1 \notin \bar{P}$.

Then for each $a$ in $P$, there exists $\mu$ in $\operatorname{Sp}(a) \cap \mathbf{R}^{+}$such that $\mu \geqq\left(r\left(a^{-1}\right)\right)^{-1}$ and $a-\mu \in \bar{P}$.

Proof. We note first that $P$ satisfies:

(iv) $a \in P, 0<t<\left(r\left(a^{-1}\right)\right)^{-1} \Rightarrow a-t \in P$.

For, given $a \in P$ and $0<t<\left(r\left(a^{-1}\right)\right)^{-1}$, we have $r\left(t a^{-1}\right)<1$ and so, by (i) and (ii), $a-t=t\left(t^{-1} a-1\right) \in P$.

Let $a \in P$, and let $\mu=\sup \left\{\lambda \in \mathbf{R}^{+}: a-\lambda \in P\right\}$. By (iv), we have $\mu \geqq\left(r\left(a^{-1}\right)\right)^{-1}>0$. Also $\mu<\infty$, for if there exists a sequence $\left\{\lambda_{n}\right\}$ with $\lambda_{n}>0, \lim _{n \rightarrow \infty} \lambda_{n}=\infty$, and $a-\lambda_{n} \in P$, then, by (i), $-1=\lim _{n \rightarrow \infty}\left(\lambda_{n}^{-1} a-1\right) \in \bar{P}$, which contradicts (iii).

It is clear that $a-\mu \in \bar{P}$, and so it only remains to prove that $\mu \in \operatorname{Sp}(a)$. Suppose, on the contrary, that $a-\mu \in \operatorname{Inv}(A)$. There exists a sequence $\left\{\lambda_{n}\right\}$ with $0<\lambda_{n} \leqq \mu, a-\lambda_{n} \in P$, and $\lim _{n \rightarrow \infty} \lambda_{n}=\mu$. By the continuity of the inverse mapping on $\operatorname{Inv}(A)$, we have $\lim _{n \rightarrow \infty}\left(a-\lambda_{n}\right)^{-1}=(a-\mu)^{-1}$, and since, on a commutative subalgebra, the spectral radius is a semi-norm dominated by the norm, $\lim _{n \rightarrow \infty} r\left(\left(a-\lambda_{n}\right)^{-1}\right)=r\left((a-\mu)^{-1}\right)>0$. Choose $t$ with

$$
0<t<\left(r\left((a-\mu)^{-1}\right)\right)^{-1} \text {. }
$$

Then, for sufficiently large $n$, we have

$$
0<t<\left(r\left(\left(a-\lambda_{n}\right)^{-1}\right)\right)^{-1},
$$

and (iv) gives $a-\lambda_{n}-t \in P$. But, for sufficiently large $n, \lambda_{n}+t>\mu$, contradicting the definition of $\mu$.

Corollary 2 (Yood). Let $Q$ be a subset of $A$ such that

(i) $a \in Q, t \geqq 0 \Rightarrow t a \in Q$,

(ii) $a \in Q, r(a)<1 \Rightarrow a(1-a)^{-1} \in Q$,

(iii) $-1 \notin \bar{Q}$.

Then $\operatorname{Sp}(a) \cap \mathbf{R}^{+}$is non-void for each a in $Q$. 
Proof. Let $P=\left\{a \in \operatorname{Inv}(A): a^{-1} \in Q\right\}$. For singular elements of $Q$ we have $0 \in \mathrm{Sp}(a)$, and so it is enough to prove that $Q \cap \operatorname{Inv}(A) \subset \Gamma$. For $a \in \operatorname{Inv}(A)$, we have $\operatorname{Sp}(a)=\left(\operatorname{Sp}\left(a^{-1}\right)\right)^{-1}$, and so it is enough to prove that $P \subset \Gamma$. Given $a \in P$ with $r\left(a^{-1}\right)<1$, we have $a^{-1} \in Q$, and therefore

$$
(a-1)^{-1}=a^{-1}\left(1-a^{-1}\right)^{-1} \in Q,
$$

$a-1 \in P$. Thus $P$ satisfies condition (ii) of Theorem 1 , and it obviously satisfies conditions (i) and (iii). Therefore $P \subset \Gamma$.

Notation. Let $\delta>0$, let $D_{\delta}=\left\{z \in \mathbf{C}:|z|>\delta^{-1}\right\}$, and let $\Phi$ be a complex valued function holomorphic on $D_{\delta}$ such that $\Phi(z)-z+1 \rightarrow 0$ as $|z| \rightarrow \infty$. Let functions $\Phi_{0}, \Phi_{1}, \Phi_{2}, \ldots$ be defined recursively by

$$
\Phi_{0}(z)=z, \quad \Phi_{k}(z)=\Phi\left(\Phi_{k-1}(z)\right) \quad(k=1,2, \ldots) .
$$

Lemma 3. For all $a \in \operatorname{Inv}(A)$ with $r\left(a^{-1}\right)<\delta, \Phi(a)$ is defined, and as $\left\|a^{-1}\right\| \rightarrow 0$.

$$
\|\Phi(a)-a+1\| \rightarrow 0
$$

Proof. Let $a \in \operatorname{Inv}(A)$ with $r\left(a^{-1}\right)<\delta$. Then $\operatorname{Sp}(a) \subset D_{\delta}$, and the functional calculus gives a well defined element $\Phi(a)$ of $A$. Since $\Phi$ is holomorphic on $D_{\delta}$, it has the Laurent series representation

$$
\Phi(z)=\sum_{n=0}^{\infty} \alpha_{n} z^{n}+\sum_{n=1}^{\infty} \beta_{n} z^{-n} \quad\left(z \in D_{\delta}\right)
$$

But $\Phi(z)-z+1-\sum_{n=1}^{\infty} \beta_{n} z^{-n} \rightarrow 0$ as $|z| \rightarrow \infty$, and so $\sum_{n=0}^{\infty} \alpha_{n} z^{n}-z+1$ is a bounded entire function that tends to zero as $|z| \rightarrow \infty$, and is therefore identically zero. Therefore

We now have

$$
\Phi(z)=z-1+\sum_{n=1}^{\infty} \beta_{n} z^{-n} \quad\left(z \in D_{\delta}\right)
$$

$$
\Phi(a)-a+1=\sum_{n=1}^{\infty} \beta_{n} a^{-n}
$$

which tends to zero in norm as $\left\|a^{-1}\right\| \rightarrow 0$.

The next lemma is an adaptation of a lemma in (1).

Lemma 4. Given $\varepsilon>0$, there exists $N$ such that, for all $n \geqq N$ and all $a \in \operatorname{Inv}(A)$ with $\left\|a^{-1}\right\|<(1+2 \varepsilon)^{-1}$, we have

(i) $\Phi_{k}(n a) \in \operatorname{Inv}(A)$ and $\left\|\left(\Phi_{k}(n a)\right)^{-1}\right\|<\delta \quad(0 \leqq k<n)$,

(ii) $\left\|n^{-1} \Phi_{n}(n a)-(a-1)\right\|<\varepsilon$.

Proof. We use the following well known elementary fact: $a \in \operatorname{Inv}(A), b \in A,\|b-a\|<\frac{1}{2}\left\|a^{-1}\right\|^{-1} \Rightarrow b \in \operatorname{Inv}(A)$ and

$$
\left\|b^{-1}\right\| \leqq 2\left\|a^{-1}\right\| \text {. }
$$


Let $\varepsilon>0$. By Lemma 3, there exists $\eta$ with $0<\eta \leqq \delta$, such that

$$
x \in \operatorname{Inv}(A),\left\|x^{-1}\right\|<\eta \Rightarrow\|\Phi(x)-x+1\|<\varepsilon .
$$

Let $a \in \operatorname{Inv}(A)$ with $\left\|a^{-1}\right\|<(1+2 \varepsilon)^{-1}$, choose a positive integer $N$ with $N>(\varepsilon \eta)^{-1}$, and let $n \geqq N$. We have

$$
\left\|(n a)^{-1}\right\|<(n+2 n \varepsilon)^{-1}<(n \varepsilon)^{-1}<\eta
$$

and so by (2), $\Phi(n a)$ is defined and

$$
\|\Phi(n a)-n a+1\|<\varepsilon
$$

We show, by induction, that

$$
\left\|\left(\Phi_{k-1}(n a)\right)^{-1}\right\|<\eta \text { and }\left\|\Phi_{k}(n a)-n a+k\right\|<k \varepsilon \quad(1 \leqq k \leqq n) .
$$

The case $k=1$ of (5) is given by (3) and (4). Suppose that (5) holds for some $k \leqq n-1$. Since $(n a-k)^{-1}=n^{-1} a^{-1}\left(1-k n^{-1} a^{-1}\right)^{-1}$, we have

$$
\begin{aligned}
\left\|(n a-k)^{-1}\right\| & \leqq n^{-1}\left\|a^{-1}\right\|\left\|\left(1-k n^{-1} a^{-1}\right)^{-1}\right\| \\
& \leqq n^{-1}\left\|a^{-1}\right\| \sum_{r=0}^{\infty} k^{r} n^{-r}\left\|a^{-1}\right\|^{r} \\
& =n^{-1}\left\|a^{-1}\right\|\left(1-k n^{-1}\left\|a^{-1}\right\|\right)^{-1} \\
& =\left\|a^{-1}\right\|\left(n-k\left\|a^{-1}\right\|\right)^{-1} \\
& <(1+2 \varepsilon)^{-1}\left(n-k(1+2 \varepsilon)^{-1}\right)^{-1} \\
& =(n(1+2 \varepsilon)-k)^{-1}, \\
\left\|(n a-k)^{-1}\right\| & <(2 n \varepsilon)^{-1}<\frac{1}{2}(k \varepsilon)^{-1} .
\end{aligned}
$$

(5) and (6) show that

$$
\left\|\Phi_{k}(n a)-(n a-k)\right\|<\frac{1}{2}\left\|(n a-k)^{-1}\right\|^{-1},
$$

and so, by (1), $\Phi_{k}(n a) \in \operatorname{Inv}(A)$, and

$$
\left\|\left(\Phi_{k}(n a)\right)^{-1}\right\|<2\left\|(n a-k)^{-1}\right\|<(n \varepsilon)^{-1}<\eta .
$$

We now take $x=\Phi_{k}(n a)$ in (2), and obtain

$$
\left\|\Phi_{k+1}(n a)-\Phi_{k}(n a)+1\right\|<\varepsilon \text {. }
$$

With (5), this gives

$$
\left\|\Phi_{k+1}(n a)-n a+k+1\right\|<(k+1) \varepsilon,
$$

and induction completes the proof of (5) for $1 \leqq k \leqq n$.

In particular, we have

$$
\left\|\Phi_{n}(n a)-n a+n\right\|<n \varepsilon,
$$

and division by $n$ gives the required inequality.

Theorem 5. Let $\delta>0$ and $D_{\delta}=\left\{z \in \mathbf{C}:|z|>\delta^{-1}\right\}$. Let $\Phi$ be holomorphic on $D_{\delta}$ and let $\Phi(z)-z+1 \rightarrow 0$ as $|z| \rightarrow \infty$. Let $P$ be a subset of $\operatorname{Inv}(A)$ such 
that

(i) $a \in P, t>0 \Rightarrow t a \in P$,

(ii) $a \in P, r\left(a^{-1}\right)<\delta \Rightarrow \Phi(a) \in P$.

Then

$$
a \in P, r\left(a^{-1}\right)<1 \Rightarrow a-1 \in \bar{P} .
$$

Proof. Let $a \in P$ with $r\left(a^{-1}\right)<1$. Among the algebra-norms on $A$ equivalent to the given norm we choose one, which we denote by $\|\cdot\|$, for which $\left\|a^{-1}\right\|<1$. Given $\varepsilon$ with $0<\varepsilon<\frac{1}{2}\left(\left\|a^{-1}\right\|^{-1}-1\right)$, we have $\left\|a^{-1}\right\|<(1+2 \varepsilon)^{-1}$. Therefore by Lemma 4 , there exists $N$ such that for all $n \geqq N, n^{-1} \Phi_{n}(n a) \in P$ and

Therefore $a-1 \in \bar{P}$.

$$
\left\|n^{-1} \Phi_{n}(n a)-(a-1)\right\|<\varepsilon \text {. }
$$

Remark. As a particular case of Theorem 5 we can take

$$
\delta=1, \Phi(z)=z^{2}(1+z)^{-1} .
$$

Thus if $P$ is a subset of $\operatorname{Inv}(A)$ satisfying

(i) $a \in P, t>0 \Rightarrow t a \in P$,

(ii) $a \in P, r\left(a^{-1}\right)<1 \Rightarrow a^{2}(1+a)^{-1} \in P$,

then

$$
a \in P, r\left(a^{-1}\right)<1 \Rightarrow a-1 \in \bar{P} .
$$

Corollary 6. Let $\delta, \Phi$ satisfy the conditions of Theorem 5 , and let $P$ be $a$ relatively closed subset of $\operatorname{Inv}(A)$ such that

(i) $a \in P, t>0 \Rightarrow t a \in P$,

(ii) $a \in P, r\left(a^{-1}\right)<\delta \Rightarrow \Phi(a) \in P$,

(iii) $-1 \notin P$.

Then for each $a \in P$, there exists $\mu \in \operatorname{Sp}(a) \cap \mathbf{R}^{+}$with $\mu \geqq\left(r\left(a^{-1}\right)\right)^{-1}$ and $a-\mu \in \bar{P}$.

Proof. If $a \in P$ with $r\left(a^{-1}\right)<1$, then $a-1 \in \operatorname{Inv}(A)$, and so, by Theorem 5 , $a-1 \in P$. Then Theorem 1 applies.

Notation. Let $\delta>0$, and let $F_{\delta}$ denote the class of complex functions $g$ holomorphic on the disc $\{z \in \mathbf{C}:|z|<\delta\}$, with no zeros in the punctured disc $\{z \in \mathbf{C}: 0<|z|<\delta\}$, and with power series expansion

with $\left|\alpha_{1}\right|>0,\left|\alpha_{2},\right|>0$.

$$
g(z)=\sum_{n=1}^{\infty} \alpha_{n} z^{n} \quad(|z|<\delta)
$$

It should be noticed that every function $g$ holomorphic in a neighbourhood of 0 and with $g(0)=0, g^{\prime}(0)>0, g^{\prime \prime}(0)>0$ belongs to the class $F_{\delta}$ for a suitable $\delta>0$. 
Theorem 7. Let $g$ belong to the class $F_{\delta}$ just defined, and let $Q$ be a subset of $A$ such that

(i) $a \in Q, t \geqq 0 \Rightarrow t a \in Q$,

(ii) $a \in Q, r(a)<\delta \Rightarrow g(a) \in Q$,

(iii) $-1 \notin \bar{Q}$.

Then $\operatorname{Sp}(a) \cap \mathbf{R}^{+}$is non-void for each a in $Q$.

Proof. We may assume $Q$ closed, by continuity of the mapping $a \rightarrow g(a)$. We may also assume that $\alpha_{1}=\alpha_{2}=1$, since the function $h$ defined by

$$
h(z)=\alpha_{2} \alpha_{1}^{-2} g\left(\alpha_{1} \alpha_{2}^{-1} z\right)
$$

belongs to $F_{\delta^{\prime}}$, where $\delta^{\prime}=\delta\left|\alpha_{2}\right|\left|\alpha_{1}\right|^{-1}$, and

$$
a \in Q, r(a)<\delta^{\prime} \Rightarrow r\left(\alpha_{1} \alpha_{2}^{-1} a\right)<\delta \Rightarrow g\left(\alpha_{1} \alpha_{2}^{-1} a\right) \in Q .
$$

We therefore assume that $g \in F_{\delta}$ and

where $f(z)=\sum_{n=3}^{\infty} \alpha_{n} z^{n}$

$$
g(z)=z+z^{2}+f(z)
$$

Let $P=\left\{a \in \operatorname{Inv}(A): a^{-1} \in Q\right\}$, and $\Phi(z)=\left(g\left(z^{-1}\right)\right)^{-1}\left(z \in D_{\delta}\right)$. We have $\Phi(z)=z^{2}\left(1+z+z^{2} f\left(z^{-1}\right)\right)^{-1}$, and it is easily verified that $\Phi, P$ satisfy the conditions of Corollary 6 . The proof is completed as in the proof of Corollary 2.

Corollary 8. Let $Q$ be a subset of $A$ such that

(i) $a \in Q, t \geqq 0 \Rightarrow t a \in Q$,

(ii) $a \in Q, r(a)<1 \Rightarrow a+a^{2} \in Q$,

(iii) $-1 \notin \bar{Q}$.

Then $\operatorname{Sp}(a) \cap \mathbf{R}^{+}$is non-void for each $a$ in $Q$.

Proof. Clear.

Remarks. It is obvious that condition (ii) in Corollary 8 can be replaced by the stronger condition:

(ii) $a \in A \Rightarrow a+a^{2} \in Q$.

An example of a set $Q$ satisfying (i), (ii) and (iii) but not (ii)' is given by the set $Q=\left\{(\alpha, \beta) \in \mathbf{C}^{2}: \alpha \in \mathbf{R}^{+},-\beta \in \mathbf{R}^{+}\right\}$in the algebra $\mathbf{C}^{2}$ (with coordinatewise multiplication).

If $0<\delta<1$, the function $g(z)=z+z^{2}$ is, a fortiori, in the class $F_{\delta}$ and so (ii) may also be replaced by the weaker condition:

(ii)" $a \in Q, r(a)<\delta \Rightarrow a+a^{2} \in Q$.

3

Given a closed set $Q \subset A$ satisfying conditions (i) and (ii) in Corollary 8 , 
an application of the Remark following Theorem 5 to the set

shows that

$$
P=\left\{a \in \operatorname{Inv}(A): a^{-1} \in Q\right\}
$$

$$
a \in Q \cap \operatorname{Inv}(A), r(a)<1 \Rightarrow a(1-a)^{-1} \in Q \text {. }
$$

In the next theorem, we show that the full force of Yood's condition holds, i.e.

$$
a \in Q, r(a)<1 \Rightarrow a(1-a)^{-1} \in Q \text {. }
$$

First we give an example to show that the reverse implication does not hold, so that this condition is not equivalent to (ii).

Example. Let $a \in \operatorname{Inv}(A) \cap \Gamma$, and let $v \in \operatorname{Sp}(a) \cap \mathbf{R}^{+}$. Clearly $v>0$. Let $P=\left\{\alpha a-\beta ; \quad \alpha>0, \quad \beta \geqq 0, \alpha^{-1} \beta<v, \quad \alpha^{-1} \beta \notin \operatorname{Sp}(a)\right\}$. Then $P \subset \operatorname{Inv}(A)$ and $x \in P, t>0 \Rightarrow t x \in P$.

Suppose now that $x \in P$ with $r\left(x^{-1}\right)<1$; i.e. $x=\alpha a-\beta$ with $\alpha>0, \beta \geqq 0$, $\alpha^{-1} \beta<v, \alpha^{-1} \beta \notin \operatorname{Sp}(a)$ and $r\left(\left(a-\alpha^{-1} \beta\right)^{-1}\right)<\alpha$. Then $\left|\lambda-\alpha^{-1} \beta\right|>\alpha^{-1}$ for all $\lambda \in \operatorname{Sp}(a)$, and in particular $v-\alpha^{-1} \beta>\alpha^{-1}$. It follows that $\alpha^{-1}(\beta+1) \notin \operatorname{Sp}(a)$ and $\alpha^{-1}(\beta+1)<v$. Thus $x-1 \in P$. We have proved that $P$ satisfies conditions (i) and (ii) of Theorem 1 .

Let $Q=\left\{x \in \operatorname{Inv}(A): x^{-1} \in P\right\} \cup\{0\}$. Then $Q$ satisfies conditions (i) and (ii) of Corollary 2. But it is clear that in general it does not satisfy condition (ii) of Corollary 8. For a concrete example (in which also $-1 \notin \bar{P}$ ), take $A=C[0,1]$ and take $a$ to be the function $a(t)=1+t$.

Suppose now that $a$ is an element of $\operatorname{Inv}(A) \cap \Gamma$ such that $\operatorname{Sp}(a) \cap \mathbf{R}^{+}$is not connected. Then we may choose $v>\left(r\left(a^{-1}\right)\right)^{-1}$ such that $v$ is the supremum of a component of the intersection of $\mathbf{R}^{+}$with the resolvent set of $a$. With this choice of $v$ and with $P$ defined as above, we have

$$
\sup \left\{\lambda \in \mathbf{R}^{+}: a-\lambda \in P\right\}=v,
$$

which shows that in Theorem 1 we may have $\mu>\left(r\left(a^{-1}\right)\right)^{-1}$.

Theorem 9. Let $Q$ be a closed subset of $A$ such that

(i) $a \in Q, t \geqq 0 \Rightarrow t a \in Q$,

(ii) $a \in Q, r(a)<1 \Rightarrow a+a^{2} \in Q$.

Then, $a \in Q, r(a)<1 \Rightarrow a(1-a)^{-1} \in Q$.

Proof. Let $a \in Q$, and consider the sequence defined by

$$
a_{1}=a, \quad a_{k}=a_{k-1}+a_{k-1}^{2} \quad k=2,3,4, \ldots
$$

Then $a_{k}$ is a polynomial in $a$ which we may write as

$$
a_{k}=\sum_{i=1}^{\infty} \mu_{i}(k) a^{i}
$$

We shall prove by induction that the coefficients $\mu_{i}(k)$ satisfy the following relations, for all $i, k \geqq 1$ :

$$
\mu_{i}(k) \leqq k^{i-1}
$$

E.M.S. $-19 / 1-E$ 


$$
\mu_{i}(k) \leqq\left(\frac{k}{k+1}\right)^{i-1} \mu_{i}(k+1) .
$$

The definition of $a_{k}$ shows that the $\mu_{i}(k)$ satisfy

$$
\mu_{i}(k)=\mu_{i}(k-1)+\sum_{j=1}^{i-1} \mu_{j}(k-1) \mu_{i-j}(k-1) .
$$

Since this last sum is empty when $i=1$, we have $\mu_{1}(k)=1$ for all $k$ and, clearly, $\mu_{i}(1)=0$ for $i \geqq 2$, so that (1) holds if either $i$ or $k$ is 1 . Now suppose that (1) has been established for all pairs $(i, k)$ with $1 \leqq i<j$ and for all $(i, k)$ with $i=j$ and $1 \leqq k<n$. We shall prove that this implies that (1) holds for the pair $(j, n)$. Then induction first on the second coordinate and then on the first coordinate proves (1) for all pairs $(i, k)$.

We have $\mu_{j}(n)=\mu_{j}(n-1)+\sum_{r=1}^{j-1} \mu_{r}(n-1) \mu_{j-r}(n-1)$ and so, by the induction hypothesis,

as required.

$$
\begin{aligned}
\mu_{j}(n) & \leqq(n-1)^{j-1}+\sum_{r=1}^{j-1}(n-1)^{r-1}(n-1)^{j-r-1} \\
& =(n-1)^{j-1}+(j-1)(n-1)^{j-2} \leqq(n-1+1)^{j-1}=n^{j-1}
\end{aligned}
$$

The proof of (2) follows the same pattern. Since $\mu_{1}(k)=\mu_{1}(k+1)=1$ and $\mu_{i}(1)=0$ for all $i \geqq 2$, the cases $k=1$ and $i=1$ are trivial. Suppose then, that (2) holds for $1 \leqq k \leqq n$ and $1 \leqq i \leqq j$ and consider $\mu_{j}(n+1)$. Then, from $\mu_{j}(n+1)-\mu_{j}(n)=\sum_{r=1}^{j-1} \mu_{r}(n) \mu_{j-r}(n)$, the induction hypothesis gives us

$$
\begin{aligned}
\mu_{j}(n+1)-\mu_{j}(n) & \geqq \sum_{r=1}^{j-1} \mu_{r}(n-1)\left(\frac{n}{n-1}\right)^{r-1} \mu_{j-r}(n-1)\left(\frac{n}{n-1}\right)^{j-r-1} \\
& =\left(\frac{n}{n-1}\right)^{j-2} \sum_{r=1}^{j-1} \mu_{r}(n-1) \mu_{j-r}(n-1) \\
& =\left(\frac{n}{n-1}\right)^{j-2}\left(\mu_{j}(n)-\mu_{j}(n-1)\right) \\
& \geqq\left(\frac{n}{n-1}\right)^{j-2}\left(1-\left(\frac{n-1}{n}\right)^{j-1}\right) \mu_{j}(n),
\end{aligned}
$$

this last inequality again by the induction hypothesis. But

$$
\left(\frac{n}{n-1}\right)^{j-2}\left(1-\left(\frac{n-1}{n}\right)^{j-1}\right)=\left(\frac{n-1}{n}\right)\left(\left(1+\frac{1}{n-1}\right)^{j-1}-1\right)
$$

and binomial expansion of the last bracket gives

$$
\left(\frac{n-1}{n}\right)\left(\left(1+\frac{1}{n-1}\right)^{j-1}-1\right) \geqq\left(1+\frac{1}{n}\right)^{j-1}-1 \text {. }
$$


Thus $\mu_{j}(n+1) \geqq\left(\frac{n+1}{n}\right)^{j-1} \mu_{j}(n)$ and (2) is also proved.

Now, for each positive integer $n$, consider the sequence $\{b(k, n)\}$ of elements of $Q$ defined by

$$
\begin{aligned}
& b(1, n)=n^{-1} a \\
& b(k, n)=b(k-1, n)+(b(k-1, n))^{2} \quad k=2,3, \ldots,
\end{aligned}
$$

and, finally, the diagonal sequence $\{n b(n, n)\}$. The element $n b(n, n)$ is again a polynomial in $a$ and, in fact,

$$
n b(n, n)=\sum_{i=1}^{\infty} n^{1-i} \mu_{i}(n) a^{i}=\sum_{i=1}^{\infty} \lambda_{i}(n) a^{i}
$$

From (1) and (2) we see that, for all $n, i$,

$$
\lambda_{i}(n) \leqq \lambda_{i}(n+1) \leqq 1 .
$$

Let $\beta_{i}=\lim _{n \rightarrow \infty} \lambda_{i}(n)$. We prove, by induction, that $\beta_{i}=1$ for all $i$. That $\beta_{1}=1$ is clear, since $\lambda_{1}(n)=1$ for all $n$. Let $i \geqq 2$ and suppose that $\beta_{j}=1(1 \leqq j<i)$. The relation (3) gives

$$
\left(\frac{n}{n-1}\right)^{i-1} \lambda_{i}(n)=\lambda_{i}(n-1)+\frac{1}{n-1} \sum_{j=1}^{i-1} \lambda_{j}(n-1) \lambda_{i-j}(n-1),
$$

which we may rearrange first as

$$
\left[\left(1+\frac{1}{n-1}\right)^{i-1}-1\right] \lambda_{i}(n)=\lambda_{i}(n-1)-\lambda_{i}(n)+\frac{1}{n-1} \sum_{j=1}^{i-1} \lambda_{j}(n-1) \lambda_{i-j}(n-1),
$$

and then as

$$
(i-1) \lambda_{i}(n)\left[1+O\left(\frac{1}{n-1}\right)\right]=(n-1)\left(\lambda_{i}(n-1)-\lambda_{i}(n)\right)+\sum_{j=1}^{i-1} \lambda_{j}(n-1) \lambda_{i-j}(n-1) .
$$

Since

$$
\lim _{n \rightarrow \infty} \sum_{j=1}^{i-1} \lambda_{j}(n-1) \lambda_{i-j}(n-1)=i-1
$$

this gives

$$
\lim _{n \rightarrow \infty}(n-1)\left(\lambda_{i}(n-1)-\lambda_{i}(n)\right)=(i-1)\left(\beta_{i}-1\right)
$$

But $\lambda_{i}(n-1)-\lambda_{i}(n) \leqq 0$ and $\sum_{n=1}^{\infty}\left(\lambda_{i}(n-1)-\lambda_{i}(n)\right)$ converges. Therefore $\beta_{i}-1=0$.

Now suppose $r(a)<1$, and let $b=a(1-a)^{-1}=\sum_{i=1}^{\infty} a^{i}$. Given $\varepsilon>0$, there 
exists $N$ such that $\left\|\sum_{i=N+1}^{\infty} a^{i}\right\|<\frac{1}{2} \varepsilon ;$ therefore we have

$$
\begin{aligned}
\|n b(n, n)-b\| & =\left\|\sum_{i=1}^{\infty}\left(\lambda_{i}(n)-1\right) a^{i}\right\| \\
& \leqq \sum_{i=1}^{N}\left|\hat{\lambda}_{i}(n)-1\right|\|a\|^{i}+\left\|\sum_{i=N+1}^{\infty} a^{i}\right\| \\
& <\frac{1}{2} \varepsilon+\frac{1}{2} \varepsilon=\varepsilon
\end{aligned}
$$

if $n$ is sufficiently large. Thus, since $n b(n, n) \in Q$ for all $n$ and $Q$ is closed, $b \in Q$.

Remark. This gives an alternative proof of Corollary 8 as a consequence of Theorem 9 and Corollary 2.

4

As applications we give the following two theorems of which the second, at least, is already known (2).

Theorem 10. Let $A$ be a Banach *-algebra with unit and let

$$
Q=\left\{x^{*} x: x \in A\right\}
$$

Then either $-1 \in \bar{Q}$ or $\operatorname{Sp}(a) \cap \mathbf{R}^{+}$is non-void for all in $Q$.

Proof. Let $a \in Q$ and let $r(a)<1$. Then $a=x^{*} x$ for some $x$ in $A$ so that $a+a^{2}=x^{*} x+x^{*} x x^{*} x=x^{*}\left(1+x x^{*}\right) x$. Now $r\left(x x^{*}\right)=r\left(x^{*} x\right)=r(a)<1$ and so, by Ford's square root lemma (3), there exists $b$ in $A$ such that $b^{*}=b$ and $1+x x^{*}=b^{2}$. Thus

$$
a+a^{2}=x^{*} b^{2} x=(b x)^{*}(b x) \in Q .
$$

It is evident that $t \geqq 0, a \in Q \Rightarrow t a \in Q$ so the result follows from Corollary 8 .

Definition. A division semi-algebra in $A$ is a set $Q \subset A$ such that $Q \neq\{0\}$ and

(i) $a \in Q, t \geqq 0 \Rightarrow t a \in Q$,

(ii) $a, b \in Q \Rightarrow a b \in Q$ and $(a+b) \in Q$,

(iii) $a \in Q, a \neq 0 \Rightarrow a \in \operatorname{Inv}(A)$ and $a^{-1} \in Q$.

Theorem 11. Let $Q$ be a closed division semi-algebra in $A$ with $-1 \notin Q$, then $Q=\mathbf{R}^{+}$.

Proof. Let $P=Q \backslash\{0\}$ and suppose $a \in P, r\left(a^{-1}\right)<1$ then

$$
\left(1-a^{-1}\right)^{-1}=\sum_{n=0}^{\infty} a^{-n}
$$

is in $Q$ ((ii) and (iii) imply $1 \in Q$ and $Q$ is closed). Since $\left(1-a^{-1}\right)^{-1}$ is in $\operatorname{Inv}(A)$ 
$\left(1-a^{-1}\right) \in P$ and hence $(a-1)=a\left(1-a^{-1}\right) \in P$. Therefore, by Theorem 1 , there exists $\mu \geqq r\left(a^{-1}\right)^{-1}$ such that $\mu \in \operatorname{Sp}(a)$ and $(a-\mu) \in \bar{P} \subset Q$. Thus $(a-\mu)$ is a singular element of $Q$ which gives $a-\mu=0$.

\section{Acknowledgements}

The authors are grateful to B. Yood for pre-publication access to (4). The second author was partially supported by National Research Council Grant A4066 and a Canada Council Leave Fellowship.

\section{REFERENCES}

(1) F. F. Bonsall, Stability theorems for cones and wedges of continuous functions. J. Functional Analysis 4 (1969), 135-145.

(2) F. F. Bonsall, Locally compact semi-algebras, Proc. London Math. Soc. (3) 13 (1963), 51-70.

(3) J. W. M. Ford, A square root lemma for Banach (*)-algebras, J. London Math. Soc. 42 (1967), 521-522.

(4) B. Yood, On non-negative spectrum in Banach algebras, Proc. Edinburgh Math. Soc. 18 (1973), 295-298.

UNIVERSITY OF EDINBURGH

EDINBURGH

AND

DALHOUSIE UNIVERSITY

Halifax, N.S. 